\title{
ITMO Photonics: center of excellence
}

\section{Anna Voznesenskaya, Vladislav Bougrov, Sergey Kozlov, Vladimir Vasilev}

Anna Voznesenskaya, Vladislav Bougrov, Sergey Kozlov, Vladimir Vasilev, "ITMO Photonics: center of excellence," Proc. SPIE 9946, Optics Education and Outreach IV, 99460V (27 September 2016); doi: 10.1117/12.2237691

EDent: SPIE Optical Engineering + Applications, 2016, San Diego, California, United States 


\title{
ITMO Photonics: Center of Excellence
}

\author{
Anna Voznesenskaya, Vladislav Bougrov, Sergey Kozlov, and Vladimir Vasilev \\ ITMO University, Russian Federation
}

\begin{abstract}
ITMO University, the leading Russian center in photonics research and education, has the mission to train highlyqualified competitive professionals able to act in conditions of fast-changing world. This paradigm is implemented through creation of a strategic academic unit ITMO Photonics, the center of excellence concentrating organizational, scientific, educational, financial, laboratory and human resources. This Center has the following features: dissemination of breakthrough scientific results in photonics such as advanced photonic materials, ultrafast optical and quantum information, laser physics, engineering and technologies, into undergraduate and graduate educational programs through including special modules into the curricula and considerable student's research and internships; transformation of the educational process in accordance with the best international educational practices, presence in the global education market in the form of joint educational programs with leading universities, i.e. those being included in the network programs of international scientific cooperation, and international accreditation of educational programs; development of mechanisms for the commercialization of innovative products - results of scientific research; securing financial sustainability of research in the field of photonics of informationcommunication systems via funding increase and the diversification of funding sources. Along with focusing on the research promotion, the Center is involved in science popularization through such projects as career guidance for high school students; interaction between student's chapters of international optical societies; invited lectures of World-famous experts in photonics; short educational programs in optics, photonics and light engineering for international students; contests, Olympics and grants for talented young researchers; social events; interactive demonstrations.
\end{abstract}

Keywords: photonics, key enabling technologies, optical education, global education, research, outreach activities, international master programs, light-based technologies, science popularization

\section{INTRODUCTION}

On December 20, 2013, the UN General Assembly 68th Session announced 2015 as the International Year of Light and Light-based technologies. In proclaiming the International Year focusing on the topic of light science and its applications, the UN has recognized the importance of raising global awareness about how photonics (light-based technologies) promote sustainable development and provide solutions to global challenges in energy, education, agriculture and health. Light plays a vital role in our daily lives and is an imperative cross-cutting discipline of science in the 21 st century.

Photonics has revolutionized medicine, opened up international communication via the Internet, and continues to be central to linking cultural, economic and political aspects of the global society. New advances in photonics will revolutionize healthcare and provide new ways of detecting, treating and even preventing illnesses. In manufacturing, laser processing will be a basic prerequisite for high-volume, low-cost manufacturing. Photonics technology will help overcome the limitations of electronics in computers through all-optical computing or even quantum computing. Photonics will move communications into the terabit era by dramatically increasing data capacity and data transmission speeds, while simultaneously reducing the networks' carbon footprint and the overall cost per bit. Photonics will play a key role in addressing the challenges of energy efficiency and moving to a lowcarbon economy. In the future, solid-state light sources are expected to outperform almost all other sources in terms 
of efficiency, offering potential energy savings of $50 \%$ or even more, when used with intelligent light management systems. Sensor applications in smart power grids, smart buildings and smart industrial process control will contribute significantly to more efficient use of resources and meeting environmental challenges. Businesses in the field of photonics and light-based technologies work on solving key societal challenges, such as energy generation and energy efficiency, healthy ageing of the population, climate change, and security.

Photonic technologies have major impact on the world and national economy. The global photonics market was valued at USD 500 billion in 2013. It is expected to increase to USD 770 billion in 2020. Growth in the photonics industry more than doubles that of the worldwide gross domestic product (GDP). Increasing demand for high speed electronics is one of the major factors fuelling the demand for photonics based equipment. Apart from this, low power consumption of photonics equipment compared to traditional instruments is also expected to boost the demand for photonics based instruments. Photonics finds application across consumer electronics, displays, safety and defense technology, communication, metrology, sensing and photovoltaic, light sources, medical and healthcare, and the high performance computing segment. Major segment of photonics applications can be generally described as photonic information-communications systems. Photonics based information-communications systems demonstrate a rapid rate of change in capabilities in terms of price/performance.

In Europe, Photonics has been selected by the European Commission as one of 6 Key Enabling Technologies (KETs). Other KETs overlapping with Photonics are Advanced Materials and Nano Technology. European Technology Platform (ETP) Photonics 21 issued the Strategic Photonics Multiannual Roadmap for 2014-2020 as the main strategy description of Photonic development in Europe [1]. ITMO University was the only Russian university invited for participation in the ETP Photonics 21 work groups.

Developing the Photonics 21 initiative and advancing the ITMO competitive program " 5 in 100 " [2] in the roadmap of ITMO University'2016 was included creation and advancement of a center of excellence "ITMO Photonics" as a core body combining all educational, research and outreach departments involved in photonic and light technologies.

ITMO University has a long and successful history in photonics research. The world pioneer of holography prof. Yu.N Denisyuk graduated from the ITMO University and then was in a close collaboration with the School for years; the first in Russia (in that time Soviet Union) laser department was established at the ITMO University. Along with holography development ITMO University is a world-known school in Optical design and engineering established by prof. V. Churilovsky and advanced by prof. M. Rusinov. Considerable earth and space investigations were implemented with developed optical devices of excellent quality.

The potential success of the ITMO Photonics center of excellence is confirmed by its unique team occupied in advanced photonics materials, with a full suite of optical, nano-, meta- material fabrication, materials science, devices and systems. It has a remarkable collaborative history, strong connection to industry. The units included in the ITMO Photonics incorporates 780 employees, of which 224 personnel academic staff (AS), 3 corresponding members of the Russian Academy of Sciences, 50 professors and about 100 teachers/researchers with PhD degree. The average age of the AS is 45 years. Average Hirsch index $(h)$ for 10 AS key persons is $h=31$ and for 30 AS leading persons is $h=26$, respectively.

\section{ITMO PHOTONICS TASKS}

The Strategic academic unit "Photonics" at ITMO University (ITMO Photonics) is focused on realizing tremendous potential for industry and society development offered by light technologies. Photonics is on its path to making the 21 st century that of the photon, as the 20th century was that of electron, and 19th was that of steam. Photonic technologies have major impact on the world and national economy. Increasing demands in overcoming the limitations of electronics in high speed communication and in introducing modern all-optical or even quantum informatics are the major factors fuelling the need for photonics based equipment. Low power consumption and low 
carbon footprint of photonics instruments compared to traditional instruments will also to boost the demand for photonics based systems.

First of all, complementary research and engineering/technological Masters' programs within ITMO Photonics is started. Consequently, number of PhD students at ITMO Photonics has to be grown up. Opportunities for start-up companies will be systematically evaluated for the various new products. Implementation of improved products in established partner companies with connections to ITMO Photonics will be realized. New innovation-oriented educational modules are planned to be introduced.

The ITMO Photonics tasks of the international level include delivering breakthrough scientific results of world level in the framework of the network of international scientific cooperation with world leading scientific and industrial centers in the field: Australian National University, Trinity College Dublin (Ireland), University of Rochester (USA), University of California, Santa-Barbara (USA), City University of Hong Kong (China), Technische Universität Berlin (Germany) in three key-directions: (1) advanced materials of photonics; (2) ultra-fast optical and quantum informatics; (3) laser systems and technologies. Bringing together world leaders in photonics and advanced photonic materials, ITMO Photonics will provide a balance of long term exploratory high risk, high reward research and midterm research with clear transition to the established and start-up industry in Russia and in the World. ITMO Photonics will develop photonics device science and technology to realize ultimate performance. As a result, the photonics research and teaching at ITMO University will enhance their excellence, and ITMO University will have a leadership positions in photonics materials and devices, photonics technology, and as a consequence in advanced materials science and modern device physics.

The ITMO Photonics tasks of the national level include providing technological independence of Russian Federation through the development and implementation in cooperation with leading industrial partners: Khrunichev State Research and Production Space Center, Vavilov State Optical Institute, Central Research Institute «Cyclone», RTI Group, Open-code LLC, competitive technologies for protected optical and quantum networks, satellite quantum communication, components of an integrated semiconductor photonics for all-optical analog-to-digital convertors, new materials for "green" energy efficient and free from harmful elements photonics, optical coherence tomography, metamaterials, new glasses for fiber lasers and high-power LEDs, laser technology for medicine and ecology. In particular, the system will be developed for ultrafast quantum communication on the side frequencies via fiber optic lines, and prototypes of quantum networks with complex topology will be built for various regions of the Russian Federation, as the elements of the future national quantum network infrastructure. National level tasks also consider development of state educational standards, requirements and model programs for teaching in Russian Federation internationally competitive professionals in the field of photonics - the future Russian industry, including training of engineers and managers for small and medium-sized businesses.

The ITMO Photonics tasks of university level include concentration of organizational, scientific, educational, financial and human resources to create a world-class school of excellence "ITMO Photonics"; transformation of the educational process in accordance with the best international educational practices and the presence in the international university education market in the form of double degree educational programs with leading universities, i.e. those being included in the network programs of international scientific cooperation; development and creation of mechanisms for the commercialization of innovative products - results of scientific research; securing financial sustainability of research in the field of photonics, information-communication systems via funding increase and the diversification of funding sources.

Thus the ITMO Photonics paradigm is developed mainly in three interrelated directions: research, education, and outreach activities. 


\section{RESEARCH IN ITMO PHOTONICS}

ITMO Photonics integrates 21 international research laboratories (Figure 1) and accomplishes research in three general topics: (1) advanced photonics materials; (2) ultrafast optical and quantum informatics; (3) laser systems and technologies.

Topic 1 on advanced photonics materials forms a basis for the themes (2) and (3). Four ITMO Photonics international research and education centers (IRECs) are concentrated on study of materials structures (including nanostructures) and light-structure interactions. The following groups of advanced photonics materials covering the spectrum from X-rays through UV, visible, and IR up to radio diapason will be processed and new synthesis technologies will be developed: new types of nanomaterials, e.g. nanowhiskers and nanoheterostructures, including those integrated with silicon substrates; hybrid quantum nanocrystals synthesized via colloidal root; nanostructured metamaterials with unusual optical properties designed for particular applications and in order to achieve exceptional functionalities; bulk materials growth techniques from liquid phase or sol-gel precursors for glassceramics photonics media and advanced crystalline oxides, e.g. $\mathrm{Ga}_{2} \mathrm{O}_{3}$. Modern materials characterization techniques are utilized to investigate new functionalities of photonics materials and structures. Numerous applications of the developed and functionalized materials contain: ultrafast and energy-efficient optical elements for information-communication systems including optical information transmitters and detectors; cubits based on doped colloidal semiconductor nanocrystals for information storage and processing of quantum information; thermo- and radiation-stable optoelectronics devices and information-communication systems; nanophotonic components created by using metamaterials or metamaterial-inspired concepts, also known as metadevices.

Topic 2 is addressed to the ultrafast optical and quantum informatics: development of methods and systems for superfast transmission of information via super short optical signals through multi-core optical fibers; development of methods and systems for wireless information transmission in $\mathrm{THz}$ spectrum range; development of ultrafast optical switches for information flow exploring the pulses with small number of oscillations; enhancement of spectral efficiency of optical-fiber lines by utilizing multiplex quantum communication schemes; integration of quantum computing systems and logic elements in the optical and $\mathrm{THz}$ communication systems. The quantum network with dynamical control will be established to connect all ITMO University buildings. Other practical realizations will include four-nods prototype quantum network for city of Kazan and International quantum line Saint Petersburg/Helsinki to connect RUNNet (Russia) and NORDNet (North Europe) science and educational networks.

Topic 3 concludes in laser physics, laser engineering, and laser technologies. Main research directions at the "Laser systems and technologies" IREC are: new methods for generation of ultrashort optical pulses of picosecond and subpicosecond range; laser system dynamics for information analysis and processing including optical neuron networks and optical switches; laser systems for optical tomography, biosensorics, and biodiagnostics including optothermal and optoacoustic convertors; development of high power ( $\mathrm{kW}$ range) periodic pulse solid state lasers with a negligible laser beam divergence. The applications of the developed laser devices and systems will include: superfast optical information-communication systems; laser location including deep space location, support of GLONASS system, detection and monitoring of space waste; ecology monitoring for the prevention of technological disasters; medical use in optical tomography and diagnostics.

The complementarity of IRECs is apparent from a valuable set of their highly-specialized skills, expertise and experience, and social use of light technologies. Experimental expertise and up-to-date equipment of the ITMO Photonics IRECs will be combined with the wide-ranging experience in the field materials characterization and the development of new classes of materials including glasses, nanocrystals, and metamaterials. Exceptional experience in modeling and testing of optoelectronic devices will provide a strong input for new systems with extreme functional properties, e.g. laser diodes, optical amplifiers, saturable absorber mirrors, etc.

Commercialization of innovations is realized through a number of ITMO University spin-offs currently having strong connections with major industrial customers, such as «Design office of applied optics» Ltd. (contracts with 
China, Russia, and Germany), «Quantum communications» Ltd. (contracts within Russia) and «Laser» Ltd (more than 500 industrial customers).

\section{EDUCATION IN ITMO PHOTONICS}

ITMO Photonics mission is directed to accumulation and synthesis of new scientific results, consequent development and advancement of innovative photonic technologies and devices. Young scientists are main drivers in this process. Talented youth involvement into the research activities is the key goal of the ITMO Photonics. In this relation focusing of academic graduate programs is required for correspondence of their context to the global level breakthrough light-based technologies as well as actual educational technologies, high-qualified faculty staff, partnership with leading research and educational world centers etc.

ITMO University is a national leader in training and education specialists in the area direction of higher education "Photonics, instrument-making, optical and biotechnical systems and technologies". ITMO University is the leading institution of the Federal Educational and Methodical Association in this area [3]. It spreads to other universities the best educational practices and technologies in the educational directions "Optical engineering", "Photonics and optoinformatics", "Laser instruments and laser technologies". ITMO University is a leader in Russian Federation for student-admission score and the number of students accepted to budget (subsidized by State) undergraduate educational programs (Figure 2). In 2015, the average score of Unified State Exam for admitted undergraduates was 77.6. For the moment, the divisions of ITMO Photonics have 1788 students that is $15 \%$ of total student number at ITMO University. The ITMO Photonics trains $238 \mathrm{PhD}$ students from total 817 those at the University that is $29 \%$. More than $60 \%$ of ITMO Photonics students are involved to the current research projects and paid for their work. Foreign students consist more than $20 \%$ of ITMO Photonics students' body. Remarkably, that $100 \%$ of graduates are employed; more than $20 \%$ of graduates enter the PhD studies.

The quality of ITMO University educational programs in the field of photonics is certified by the international accreditation agency EUR-ACE and the Association of Engineering Education of Russia [4]. Seven master's degree programs were accredited in 2014 and 2015: "Applied optics", "Devices for research and modification of materials at the micro- and nanoscale level" , "Metamaterials" , "Nanomaterials and nanotechnologies for photonics and optoinformatics" , "Optics of nanostructures". There are educational programs implemented in partnership with the leading Russian and foreign international scientific and educational centers. A good example of such a program is the Double-degree master program "Optics, photonics and optoinformatics", implemented in 2013 jointly by the ITMO University (headed by S.A Kozlov) and one of the world leaders in the field of photonics University of Rochester, USA (headed by X.-Ch. Zhang); the program provides reception of two diplomas of Russian and US ones. Among other ITMO Photonics network master programs are "Molecular bio-, nano- photonics", "Engineering nanophotonics" (with University ENS de Cachan, France), "Optical design" (with the Warsaw University of Technology, Poland), "Optical engineering” (with the Changchun University of Science and Technology, China), "Physics and technology of nanostructures» (with Aalto University, Finland), etc.

In the short-time prospective ITMO Photonics is planning to advance the following initiatives in education: development of own educational standards for undergraduate and graduate programs; renewal and actualization of educational programs taking into account requirements of professional standards, recommendations of the Photonics 21 roadmap, and special forecasts of global job market trends. 


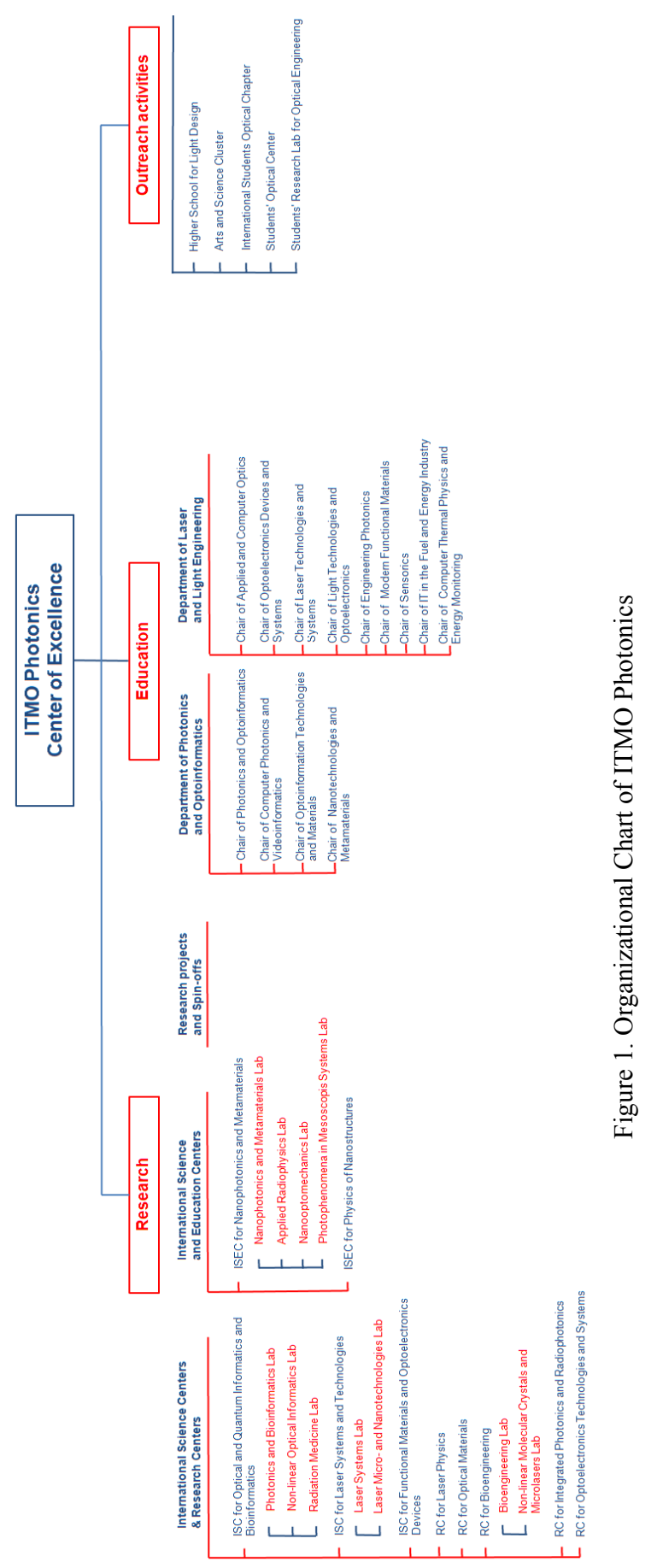

Proc. of SPIE Vol. $994699460 \mathrm{~V}-6$ 


\begin{abstract}
$\bigcirc 2013 ; 230$

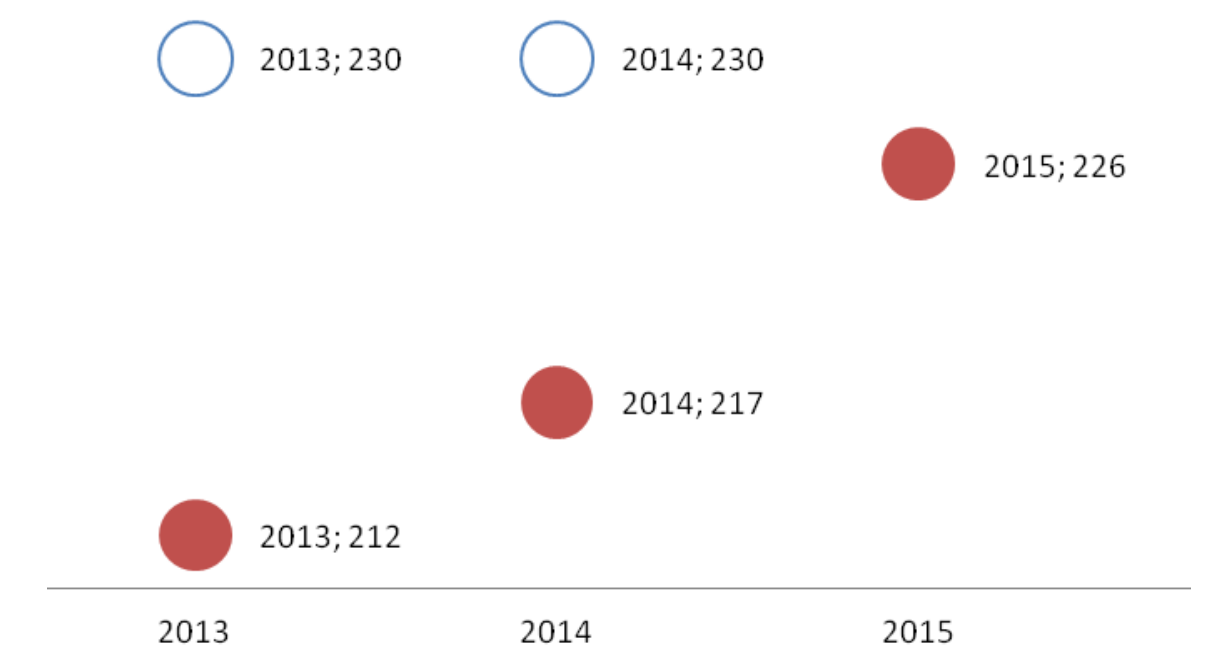

$2014 ; 230$

$2015 ; 234$

Figure 2. ITMO Photonics chart of applicants for undergraduate programs: o - average score; $\bullet$ - minimum score
\end{abstract}

\title{
5. ITMO PHOTONICS OUTREACH ACTIVITIES
}

Sustainable research and education require infrastructure focusing - development and popularization of Photonics subject area. According to the Photonics 21 roadmap [1] the dissemination of photonic technologies concludes in the optical education advancement at all levels, increase of the awareness and interest of the population to photonic technologies, support of innovations, further economic growth and expansion of the job market.

Among key points of the Photonics popularization strategy in ITMO University are: realization of short-term educational projects, coordination and support of social events and projects connected with photonic technologies; involvement experts from related areas in the popularization (school teachers, PR managers etc.); cooperation with local and international societies aiming to promote projects; wide involvement of students; developments of contests and grant programs for talented youth engaged into research and project activities; photonic installation in places of a mass population congestion (airports, railway stations, malls, parks etc.); development of interactive exhibitions with emphasis more on future technology than on historical heritage; extensive use of mass media to inform about ongoing projects, etc.

Promotional projects of the ITMO Photonics include the Higher School of Light Design and Art \& Science Cluster (Figure 1). The considerable part of the ITMO Photonics activities are student laboratories and organizations, such as the SPIE optical student chapter, School of laser technologies, and Student scientific laboratory of optics. A lot of annual and regular scientific and social events are organized and dedicated to students and youth - National Olympics in Optical engineering, Russian congress for young scientists, international events like "Optics-XXI century" Congress, "FLAMN" Congress, "Lasers and Photonics" Congress etc., International Optical Seminar, world-class interactive exhibition "Magic of Light", city festivals "GeekPicnic", "Open University", "EasyFest", etc. Besides, Optical FabLab is one of the most promising students' innovation projects of the ITMO Photonics. 


\section{CONCLUSION}

Scientific, educational and infrastructure resources were integrated in the ITMO Photonics center of excellence. This initiative is very timely and in the agreement with modern worldwide trends in photonics. The outlined three directions, namely, advanced photonics materials, ultrafast optical and quantum informatics, and laser systems clearly reflect the main strengths of the initiative. Conduction of research and teaching in the framework of the ITMO Photonics will strongly enhance its leadership in Russia and international reputation in photonics materials and devices and photonics technology upon the realization of the project.

\section{REFERENCES}

[1] Photonics Strategic Multiannual Roadmap/ http://www.photonics21.org/download/Brochures/Photonics_Roadmap_final_lowres.pdf.

[2] ITMO University competitive program " 5 in 100 ” $\mathrm{http}: / / 5100$.ifmo.ru/file/pages/9/programma.pdf.

[3] Federal Educational and Methodical Association in photonics, instrument-making, optical and biotechnical systems and technologies umo.ifmo.ru.

[4] European Network for Accreditation in Engineering Education http://www.enaee.eu/eur-ace-system/. 